\title{
Health literacy in solid-organ transplantation: a model to improve understanding
}

This article was published in the following Dove Press journal:

Patient Preference and Adherence

\author{
Marie A Chisholm-Burns' \\ Christina A Spivey ${ }^{2}$ \\ Logan R Pickett ${ }^{\prime}$
}

'University of Tennessee Health Science Center College of Pharmacy, Memphis, TN, USA; ' 2 University of Tennessee Health Science Center College of Pharmacy, Department of Clinical Pharmacy and Translational

Science, Memphis, TN, USA
Correspondence: Marie A

Chisholm-Burns

University of Tennessee Health Science

Center, College of Pharmacy, 88।

Madison Avenue, Suite 264, Memphis,

TN 38163, USA

$\mathrm{Tel}+\mid$ 90| $4487|4|$

Fax + I 90I 4487053

Email mchisho3@uthsc.edu

\begin{abstract}
Optimizing wellness and health are the most critical goals for patients post solid-organ transplantation. Low health literacy has important implications for wellness and health, increasing patient risk for negative health outcomes. More than $30 \%$ of the general US patient population has low health literacy, and solid-organ transplant recipients (SOTRs) may be especially vulnerable to low health literacy and its adverse impact on health outcomes. A comprehensive literature review was conducted and a model was adapted to better depict factors associated with low health literacy. Based on the Paasche-Orlow and Wolf model of health literacy, the Health Literacy Model in Transplantation (HeaL-T) provides a foundation to visually demonstrate the relationships among variables associated with low health literacy and to develop evidence-based strategies to improve care. The model depicts a number of patient and healthcare level factors associated with health literacy, several of which have bi-directional or reciprocal relationships, including access and utilization of healthcare, provider-patient interaction, and self-management/adherence. The impact of these factors and their relationships to SOTR outcomes are reviewed. The HeaL-T represents an important step in developing holistic understanding of the complexity of health literacy in SOTRs and offers clinicians a base from which to design strategies to mitigate adverse health effects including increased hospitalizations, graft failure, and mortality.
\end{abstract}

Keywords: health literacy, health outcomes, healthcare access, interventions, medication adherence, solid-organ transplantation

\section{Introduction}

Health literacy is defined by the Centers for Disease Control and Prevention as "the degree to which an individual has the capacity to obtain, communicate, process, and understand basic health information and services to make appropriate health decisions." The World Health Organization further defines health literacy as "cognitive and social skills which determine the motivation and ability of individuals to gain access to, understand, and use information in ways which promote and maintain good health." ${ }^{2}$ Health literacy includes the ability to read and understand medical information; properly complete medical forms; interpret numbers such as medication dosage and lab values; communicate effectively with providers; and properly adhere to medication regimens. ${ }^{3,4}$ Additionally, consideration of health literacy should encompass the task an individual is being asked to accomplish and the complexity of that task. Several measures have been developed to gage health literacy status, for example the Test of Functional Health Literacy in Adults (TOFHLA), Newest Vital Sign (NVS), and Rapid Estimate of Adult Literacy in Medicine (REALM). Basic or below basic health literacy (referred to as low health literacy) has been noted in as much as $36 \%$ 
of adults living in the United States, with intermediate health literacy in $53 \%$ and proficient health literacy in only $12 \% .{ }^{5}$

Among solid-organ transplant recipients (SOTRs), studies have noted low health literacy in anywhere from 9\% to $72 \% .^{4,6-10}$ Moreover, health literacy varies depending on factors such as instrumentation, geographic location/environment, and patient characteristics. For example, Chisholm et al found low health literacy, as measured by a modified version of the TOFHLA, in $72 \%$ of adult renal transplant recipients served by an outpatient transplant clinic. ${ }^{4}$ Using the Subjective Health Literacy Screener, Cajita et al reported a low health literacy rate of $\sim 33 \%$ in international, multicenter heart transplant recipients. ${ }^{9}$ Serper et al found low health literacy, as measured by the NVS, in 15\% of 105 liver transplant recipients at transplant centers in the Midwestern and Southern United States. ${ }^{10}$ A large study from the United Kingdom noted low health literacy in $20 \%$ of dialysis patients $(n=2,621), 15 \%$ in those wait-listed for transplant $(n=1,959)$, and $12 \%$ in patients who underwent renal transplantation $(n=2,262) .{ }^{8}$ The substantive percentage of pre- and posttransplant populations with low health literacy is concerning given noted associations between health literacy, health outcomes, and increased costs of medical care. ${ }^{3,11-14}$ Friedland reported that low health literacy in the US is associated with $\$ 73$ billion more spent on healthcare each year. ${ }^{12}$ Weiss and Palmer found that patients with low health literacy spend four times as much on healthcare each year when compared to those with better health literacy. ${ }^{13}$

Although the literature is clear on the detrimental impact of low health literacy on posttransplant outcomes (described further in the following section), a gap exists regarding a comprehensive, yet contained, foundational model that may be applied to develop interventions that mitigate the effects of low health literacy. To better depict the spectrum of factors and outcomes associated with health literacy in SOTRs, the purpose of this review was to build on the previous work of Paasche-Orlow and Wolf, and propose the adapted Health Literacy Model in Transplantation or HeaL-T (Figure 1). ${ }^{15,16}$ A further objective was to use the HeaL-T as a platform from which to briefly discuss interventions, strategies, and tools to address low health literacy in SOTRs. (Studies from both the general patient population and transplant population were

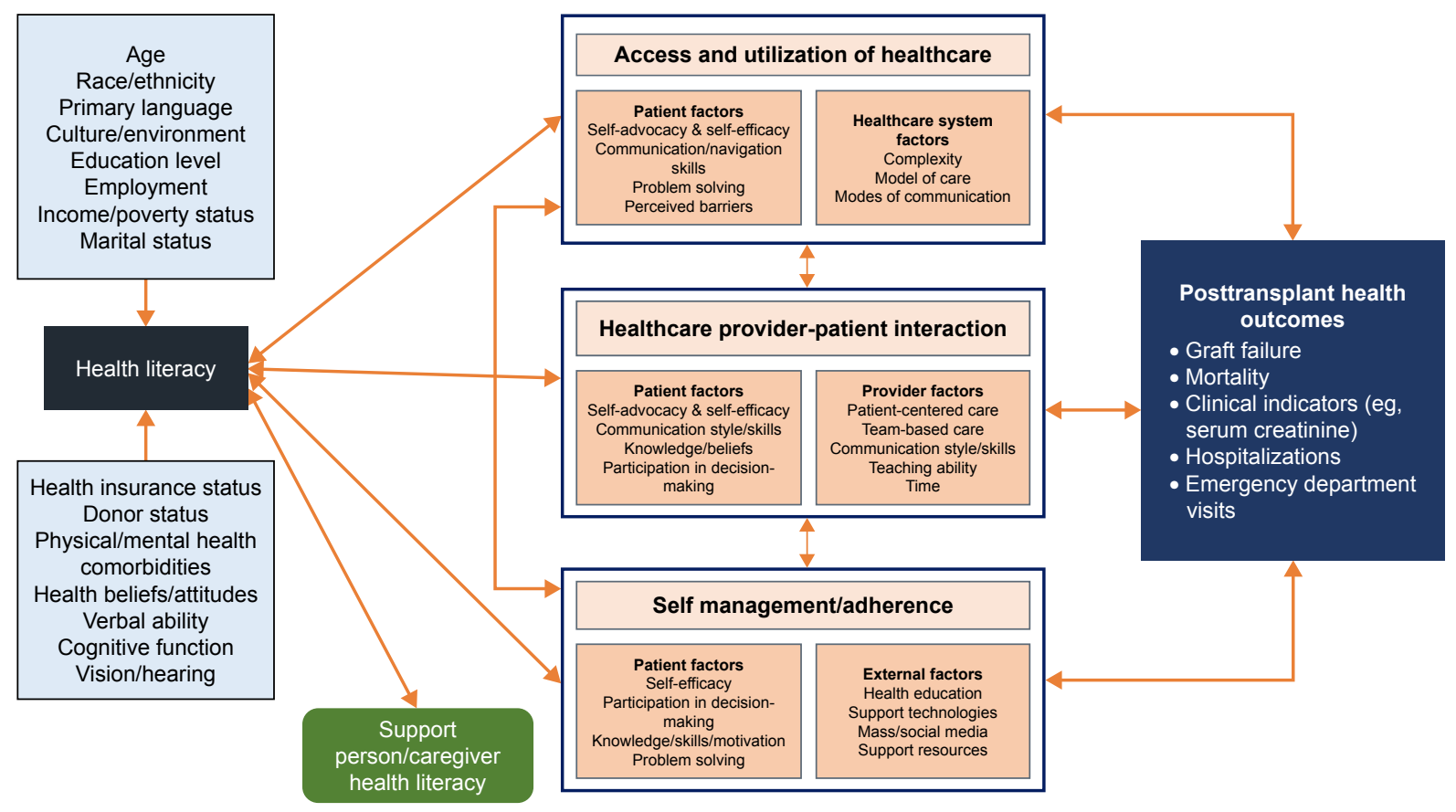

Figure I Health literacy model in transplantation (HeaL-T): patient-level factors, healthcare-level factors, and outcomes associated with health literacy in the solid-organ transplant population. ${ }^{\mathrm{a}, 15,16}$

Notes: Health literacy is associated with several patient-level (eg, age, cognitive function) and caregiver factors. Health literacy also influences, and is influenced by, caregiver and healthcare-level factors including access and utilization of healthcare, provider-patient interaction, and self-management/adherence. These factors may also influence each other; for example, a patient's adherence may be influenced by an interaction with a provider. Health outcomes are affected by all of these factors that stem from health literacy, and these outcomes can also influence healthcare-level factors as well as health literacy. For example, a patient who does not understand how to take his/her medication (health literacy), and is thus nonadherent (healthcare-level factor), is hospitalized (outcome). During the hospitalization (outcome), the patient has an effective educational interaction with a provider (healthcare-level factor) and develops an understanding of why and how to take his/her medication (health literacy). Going forward, the patient's adherence increases and his/her outcomes improve. The HeaL-T is adapted with permission from Paasche-Orlow MK, Wolf MS. The causal pathways linking health literacy to health outcomes. Am J Health Behav. 2007;3I(Suppl I):SI9-S26. ${ }^{15}$ aStudies from both the general population and transplant population were reviewed, particularly when literature in the transplant population was scarce. 
reviewed to describe the factors and relationships depicted as part of the HeaL-T, particularly when literature in the transplant population was scarce.)

\section{Posttransplant outcomes affected by health literacy}

Low health literacy is consistently associated with negative patient outcomes in the kidney disease and transplant literature. For example, prior studies among kidney transplant recipients found that lower health literacy was associated with increased serum creatinine levels, an indicator of renal dysfunction. ${ }^{7,17}$ In a recent systematic review of health outcomes and health literacy in chronic kidney disease patients (4,367 non-dialysis, 13,202 dialysis, 390 transplant, and 341 unspecified), low health literacy was associated with increased risk of mortality, emergency department use, and hospitalization. ${ }^{14}$ A study of liver transplant recipients conducted by Serper et al noted that increased treatment knowledge, which was associated with better health literacy, resulted in a $15 \%$ reduction in posttransplant hospitalization (incidence rate ratio $[\mathrm{IRR}]=0.85,95 \% \mathrm{CI}=0.74-0.97) .{ }^{10}$ Serper et al also found that low health literacy was associated with medication tradeoffs (defined as choosing between "spending money on medications vs other essential expenses") among kidney and liver transplant recipients, which in turn increased risk of hospitalization (relative risk $[\mathrm{RR}]=1.64,95 \% \mathrm{CI}=1.14-2.35) .{ }^{18}$ Miller-Matero et al reported that SOTRs with low health literacy were more likely to be readmitted to hospital after transplant as well as experience graft failure. ${ }^{19}$ Finally, and perhaps most alarmingly, Cavanaugh et al found that end-stage renal disease patients with limited health literacy had higher mortality risk than those with adequate health literacy $(\mathrm{HR}=1.54,95 \%$ $\mathrm{CI}=1.01-2.36) .{ }^{20}$ See Figure 1 for a summary of posttransplant outcomes associated with health literacy.

\section{Patient-level factors associated with health literacy}

Patient-level variables depicted in the HeaL-T (Figure 1 and Table 1) and explored in the following sections represent both modifiable and non-modifiable factors associated with health literacy. Improved understanding of these factors may assist healthcare professionals in identifying SOTRs at high risk for low health literacy. Interventions are unlikely to directly impact most patient-level factors, particularly non-modifiable demographics such age and race. However, healthcare providers and organizations can promote practices that optimize outcomes in patients with low health
Table I Relationships between patient-level factors and health literacy

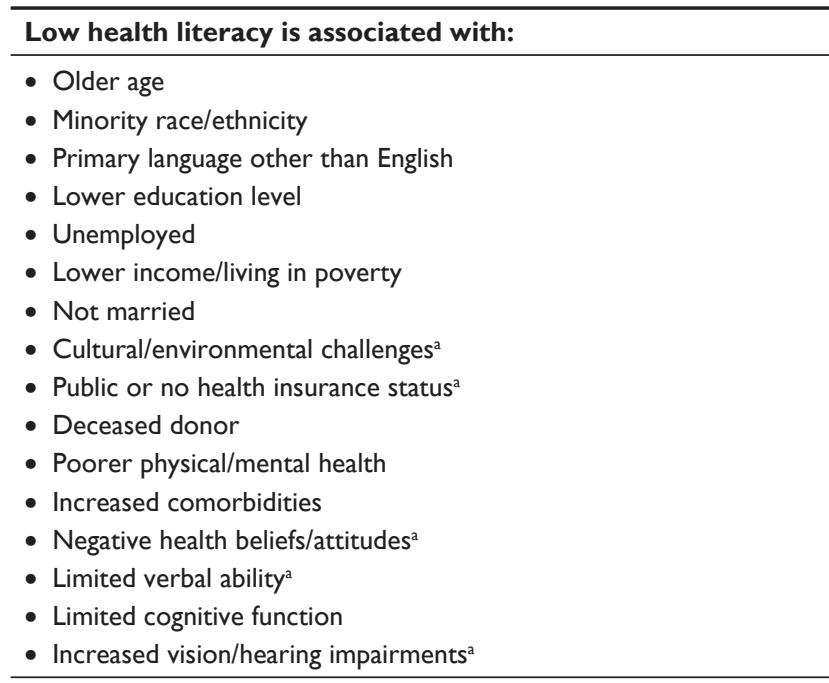

Note: association with health literacy primarily based on reviews of studies in the general patient population when transplant studies were scarce or non-existent.

literacy and close the gap (disparities) that exist across these factors.

Although addressed individually in this review, it should be remembered that patient factors often intersect and exist within a broader context that influences health literacy and, in turn, posttransplant functioning. Often one variable, such as advanced age, may serve as a proxy or signifier for an underlying issue, such as cognitive deterioration. Exploring various intersections is beyond the scope of this review, but they should be considered and evaluated as part of health literacy assessment.

\section{Age and race/ethnicity}

In general, older age has consistently been associated with low health literacy, and some studies describe those 65 years and older as being particularly affected., ${ }^{4,521-26}$ Regarding race/ethnicity, multiple US studies have demonstrated a consistent pattern of low health literacy among racial/ ethnic minorities including Hispanic and African American groups. ${ }^{5,7,20,21,23,27,28}$ In contrast, Whites and Asian/Pacific Islanders were found to have higher rates of adequate health literacy. ${ }^{5,17,23}$ For example Miller-Matero et al, in their study of health literacy and cognitive function in patients evaluated for transplant, found that White patients $(n=260)$ had higher health-related reading ability than Black patients $(n=94) .{ }^{27}$

\section{Language}

Among US SOTRs, English as primary language has been linked to health literacy. Kidney transplant recipients who 
speak English as their primary language have higher health literacy than those who do not. ${ }^{17}$ Lower health literacy is also seen in both renal failure and kidney transplant patients who primarily speak Spanish. ${ }^{6}$ Similar findings are reported in the general patient population. As noted in the National Assessment of Adult Literacy, speaking only English in the home before starting school is associated with higher health literacy than speaking other languages, or other languages plus English. ${ }^{5}$

\section{Culture and environment}

In addition to primary language, various aspects of an individual's culture may play a role in health literacy and influence the ability to understand and follow instructions of healthcare providers. ${ }^{29}$ These aspects include but are not limited to: ${ }^{29-31}$

1. Health beliefs

2. Religion/spirituality

3. Familism vs individualism, defined as prioritizing family vs the individual

4. Time orientation, defined as a cultural preference for thinking with a focus on the past, present, or future

5. High context (culture emphasizes developing deeper and longer-term group connections that facilitate implicit communication and nonverbal cues) vs low context (culture in which relationships/connections are often more superficial and task- or goal-oriented, with greater emphasis on explicit communication).

According to Shaw et al, "cultural variation can be seen in beliefs about disease etiology, appropriate treatments, proper self-care and preventive treatment, human physiology, and appropriate doctor and patient conduct." ${ }^{32}$ Singleton and Krause further explain that "patients from cultural minority groups may be more subjected to the effects of low health literacy than patients from the dominant culture because of interactions between literacy, cross-cultural communication barriers including language, and the experience of bias." 29 For example, patients may experience lack of trust in healthcare organizations and professionals due to historical patterns of mistreatment. ${ }^{29,33}$ Likewise, they may be overly trustful or deferential to healthcare organizations and/or professionals, viewing the organization's brand/reputation as equivalent to optimal patient care and the healthcare professional as the "expert"; as a result, patients may not actively participate in their own healthcare. Patients may also wish to avoid conflicts with healthcare professionals as "authority" figures and therefore may not fully engage with providers.

Prior studies suggest that cultural differences may affect knowledge, access, and utilization of healthcare services. ${ }^{32,33}$
In a well-documented example, immigrant women (particularly those from Asian backgrounds) were less likely than native-born women to utilize cervical cancer screening services; further, increased mainstream acculturation was associated with better reproductive health knowledge. ${ }^{34-37}$ Identified barriers to care in these studies included lack of: disease state and screening knowledge/awareness, female healthcare professionals, and culturally sensitive interventions. Cultural background and beliefs may also influence care options such as receiving blood transfusions as well as various aspects of transplantation such as willingness to participate in deceased and/or living organ donation. ${ }^{38}$ Jernigan et al, in a qualitative study among American Indian/ Alaska Native college students, found that many participants supported organ donation though it conflicted with cultural beliefs regarding burial. ${ }^{39}$ However, participants also expressed mistrust of the healthcare system, which may act as a barrier to donation. Transplant healthcare providers should therefore be conscious of how culture may affect not only the health literacy levels of transplant candidates, recipients, and potential donors, but also how these individuals approach and interact with the healthcare system.

Like culture, environmental factors may also be related to health literacy levels. ${ }^{40,41}$ These factors are wide-ranging and examples include public policies, health promotion programs, neighborhood crime rates, education system, media exposure, social media, and pollution. ${ }^{40}$ Although currently lacking, future research should consider the relationship between environmental factors and health literacy in the transplant population.

\section{Socioeconomic factors}

Socioeconomic factors, namely income/poverty status, employment, and education level, play a role in health literacy. Gordon and Wolf noted an association between low income and low health literacy in renal transplant recipients. ${ }^{7}$ Regarding employment status, Taylor et al found that patients who were on dialysis, wait-listed for transplantation, or receiving kidney transplantation all had lower health literacy if they were unemployed. ${ }^{8}$ Cajita et al also found that unemployment was associated with low health literacy in heart transplant recipients. ${ }^{9}$

Given the logical link between education and health literacy status, it is unsurprising that the literature examining this factor is extensive and definitive. In patients referred for transplant, both limited health-related reading ability and health-related math ability were associated with fewer years of education. ${ }^{27}$ This is consistent with Dageforde et al who noted that renal transplant recipients $(n=255)$ 
with fewer years of education were more likely to have lower health literacy $(\mathrm{OR}=0.768,95 \% \mathrm{CI}=0.669-0.882) .{ }^{22}$ Escobedo and Weismuller found that among a small sample of dialysis patients and renal transplant recipients $(n=44)$, $100 \%$ of patients with an elementary or middle school-level education and approximately $53 \%$ of patients with a high school-level education were classified as "high likelihood" or "possibility" of limited (low) health literacy compared to $\sim 21 \%$ of patients with a college-level education. ${ }^{6}$

The literature is thus consistent that transplant/chronic kidney disease patients with greater socioeconomic vulnerability - unemployed, lower income/living in poverty, and lower education level - are at greater risk of low health literacy.

\section{Health insurance status}

Literature examining the relationship between health insurance status and health literacy in the transplant population is scarce-to-nonexistent, but studies in the general patient population are informative. Sarangarm et al found that individuals with government insurance were more likely to have low health literacy compared to those with private insurance. ${ }^{42}$ The National Assessment of Adult Literacy similarly found that patients who had private or militaryprovided health insurance had higher health literacy than patients who received Medicare/Medicaid or were uninsured. ${ }^{5}$ Levy and Janke also found what they described as a "marginally" significant $(P=0.079)$ trend suggesting that adults aged 50 years or older with low health literacy were more likely to be uninsured. ${ }^{23}$ In contrast, Baker et al found that patients with Medicare or private insurance were more likely to have low health literacy when compared to patients on Medicaid or patients with no insurance. ${ }^{21}$ Based on a preponderance of the available evidence, the literature regarding health insurance status and how it affects health literacy is suggestive that public insurance or lack of insurance may be linked with lower health literacy than private insurance. Further study is warranted, especially in the transplant population.

\section{Donor status}

Although the body of literature examining donor status and its effect on health literacy status is small, the existing research suggests deceased donor status may be associated with low health literacy. In a study of kidney transplant recipients and donors $(n=360)$, Dageforde et al found that while $10 \%$ overall had low health literacy, $6 \%$ of living donors and $9 \%$ of living donor recipients had low health literacy compared to $14 \%$ of deceased donor recipients. ${ }^{22}$ Taylor et al also found that recipients of deceased donor transplantation had significantly higher prevalence of low health literacy than living donor recipients. ${ }^{8}$

We postulate the association between health literacy and donor status may be due to the more exhaustive and complex evaluative process involved with living donation. As Dageforde et al explain, consent documents for living donation are generally written at a college freshman level and difficult to navigate without assistance and/or a higher literacy level. ${ }^{22}$ The investigators further state "low and moderate health literacy transplant candidates may recruit potential donors with a similar level of health literacy who in turn have challenges navigating and completing the donation process." 22 The implication: candidates with low health literacy select living donors unable to complete the donation process, and are thus left to accept deceased donor transplants.

\section{Comorbidities/mental and physical health}

Effects of the patient's underlying disease state may impact his/her ability to understand and process information, communicate, and participate in treatment. Multiple studies also support that physical/mental health status as well as comorbidities may be related to health literacy among end-stage disease patients and SOTRs. In the end-stage renal disease population, Cavanaugh et al found that patients with a lower serum albumin were more likely to have low health literacy. ${ }^{20}$ Additionally, Taylor et al found that in dialysis patients and transplant recipients, a higher number of comorbidities, depression, and psychosis were associated with lower health literacy. ${ }^{8}$ The findings regarding comorbidities and psychosis were similar in patients who were wait-listed for transplant, though depression was not related to lower health literacy for those wait-listed. ${ }^{8}$ In a later systematic review, Taylor et al also noted lower health literacy in chronic kidney disease patients with higher blood pressure and atherosclerotic events. ${ }^{14}$ Specifically in the transplant population, Cajita et al found that lower level of physical activity was associated with lower health literacy among heart transplant recipients, ${ }^{9}$ and Demian et al reported lower health literacy in renal transplant recipients with higher levels of depression. ${ }^{17}$

\section{Health beliefs and attitudes}

Several studies in chronic disease state populations have established a link between low health literacy and erroneous or suboptimal health and/or medication beliefs and attitudes. ${ }^{43-48}$ For example, asthma and chronic obstructive pulmonary disease patients with low health literacy were more likely to express erroneous beliefs, misconceptions, or concerns about their 
illness and/or related medications. ${ }^{44-46}$ Soones et al and Kale et al noted a link between low health literacy, illness/medication beliefs and concerns, and medication nonadherence, and recommended developing interventions to address both low health literacy and disease/medication beliefs to facilitate better medication adherence. ${ }^{44,46}$ In one of the few available studies in transplant, Jones et al found that among patients wait-listed for kidney transplant ( $n=41)$, adequate health literacy was associated with strong beliefs concerning the necessity of medications and greater confidence about taking posttransplant medications. ${ }^{49}$ The existing evidence suggests an important relationship between health literacy and health beliefs that, in turn, has a considerable effect on self-management behaviors such as medication adherence (discussed later).

\section{Verbal ability, cognitive functioning, and vision/hearing}

Health literacy may be affected by patient verbal ability, cognitive functioning, and/or vision/hearing acuity, particularly in older adults..$^{23,50,51}$ In the context of health literacy, verbal ability refers to the "ability to speak and listen that facilitates exchanging, understanding, and interpreting of health information for health-decision making, disease management, and navigation of the healthcare system." 52 In a sample of older adults aged 55-74 years, Serper et al found that limited cognitive function, including verbal ability, was significantly associated with lower health literacy. ${ }^{53}$ The findings of Wolf et al were similar. ${ }^{54}$ In addition to verbal ability, cognitive functions include memory, problem-solving, reasoning, mental flexibility, the ability to learn new information, and computing, among other skills and processes needed for SOTRs to fully comprehend and participate in their own healthcare. Issues with cognitive functioning may be exacerbated by vision and/or hearing impairments. ${ }^{50,51}$ Speros suggested that visual/hearing limitations may compromise an individual's ability to fully understand or grasp information provided by the healthcare professional. ${ }^{50}$ Wallhagen et al also reported that visual and hearing impairments have significant negative effects on functioning (eg, physical abilities, mental health) among adults ages 50 years and older, which in turn may impact health literacy. ${ }^{55}$

Previous research suggests that cognitive limitations are a concern in the transplant population. For example, Bürker et al found that almost $40 \%$ of a sample of adult heart transplant recipients displayed cognitive impairments, as did more than $70 \%$ of Cohen et al's sample of lung transplant recipients. ${ }^{56,57}$ Miller-Matero et al found that limited reading ability was associated with cognitive impairment among patients referred for transplant. ${ }^{27}$ Additionally, Patzer et al reported that both lower health literacy and greater cognitive impairment were significantly associated with more limited treatment knowledge in kidney transplant recipients. ${ }^{58}$

\section{Marital status}

Although Toçi et al found no significant differences between health literacy levels in married adults vs single adults, ${ }^{25}$ other studies specifically among SOTRs disagree. ${ }^{7,17}$ For example, in renal transplant recipients, Demian et al found that being married was associated with higher health literacy, ${ }^{17}$ while Gordon and Wolf found that being single was associated with lower health literacy. ${ }^{7}$ Marital status and how it affects health literacy thus warrants consideration in the transplant population. Marital status should also be examined in the context of support person/caregiver health literacy (discussed in the following section), as spouses/partners often serve as patients' caregivers.

\section{Support person/caregiver health literacy}

Thus far we have focused our discussion on patient-level factors associated with health literacy. However, as suggested by Dageforde and Cavanaugh, comprehensive assessment of an SOTR's health literacy should also consider the health literacy level of their support person/caregiver (as applicable). ${ }^{16}$ Caregivers provide critical support and assistance to patients with chronic medical conditions such as transplantation, including self-management support, "accessing/understanding health information, communicating with healthcare providers, coordinating support services, and participating in health decision-making and problem-solving." 59 As an example, Chisholm-Burns et al found a significant, positive relationship between social support (which shares aspects of caregiving) and immunosuppressant therapy adherence among adult renal transplant recipients. ${ }^{60}$ For these reasons, support person/caregiver health literacy was included in the HeaL-T (Figure 1). Of concern, previous studies have reported that caregivers' health literacy and/or information needs regarding patient condition and treatment may be overlooked or marginalized by healthcare practitioners, even though the relationship between patient and caregiver plays an important role in patient outcomes. ${ }^{61-64}$

A systematic review conducted by Yuen et al found that low health literacy in caregivers of patients with chronic conditions may be as high as $52 \% .{ }^{59}$ Other studies have found variable levels of literacy among caregiver-patient dyads. Patients and caregivers may influence each other other's 
health literacy, whether through exchange of information, lack of this exchange, or miscommunication; the HeaL-T notes this bi-directional relationship. In a study of elderly Hispanic patients and their caregivers, Garcia et al found that the largest percentage of dyads, $41 \%$, consisted of patients with low health literacy and caregivers with adequate health literacy, while $7 \%$ of dyads had patients with adequate health literacy but caregivers with low health literacy. ${ }^{28}$ Among dyads in which patients and caregivers had the same level of literacy, 28\% had adequate health literacy and 24\% had low health literacy. ${ }^{28}$ In a study of Italian patients with heart failure and their caregivers, Della Pelle et al found that most caregivers had adequate levels of health literacy, but those who were older and had lower education levels had the lowest health literacy. ${ }^{65}$

Findings from the Yuen et al systematic review further suggest that low health literacy in caregivers is associated with increased caregiver burden, poorer patient self-management, and increased use of health services. ${ }^{59}$ The implication is that caregiver-provided support and assistance to patients may be seriously hindered by low health literacy. As there is a dearth of studies on support person/caregiver health literacy in the transplant literature, additional research is needed to more fully articulate and address the: 1) extent of low health literacy in caregivers of SOTRs; and 2) effects of support person/caregiver health literacy on transplant outcomes.

\section{Healthcare-level factors associated with health literacy Access and utilization of healthcare}

Patients with low health literacy may have difficulty interfacing with health systems in a variety of ways: identifying appropriate healthcare professionals, scheduling appointments for various medical tests, and arranging payment for treatment, among others. Likewise, the health system's infrastructure, organization, and modes of communication may not accommodate the needs of patients with varying levels of health literacy. Owing to issues such as health system complexity, communication and cognitive barriers, and medical condition(s) and overall health, patients in the general population are at risk of decreased healthcare access and inappropriate or inadequate utilization of healthcare services due to low health literacy. $23,24,34,35,66,67$

Consistent with this pattern, several studies provide significant evidence that low health literacy may severely hamper access to transplant. In an assessment of health literacy status in advanced kidney disease patients, Taylor et al noted that a lower percentage $(12 \%)$ of patients in the transplant group had low health literacy than in the wait-listed group (15\%) and dialysis group (20\%), suggesting patients with lower health literacy may be less likely to advance to renal transplantation. ${ }^{8}$ Taylor et al also conducted a systematic review examining health literacy and outcomes in chronic renal disease and found that longer time to transplantation referral was linked to low health literacy. ${ }^{14}$ Likewise, Grubbs et al, in a study of health literacy and access to renal transplantation in hemodialysis patients aged 18-75 years $(n=62)$, found that patients with low health literacy were less likely to be referred for transplant evaluation than those with adequate health literacy. ${ }^{68}$ Miller-Matero et al demonstrated that patients with lower health literacy and impaired cognitive ability were less likely to be listed for transplant and more likely to be removed from listing compared to patients who had adequate health literacy and cognitive function. ${ }^{19}$ Additionally, Kazley et al indicated that lower health literacy was significantly associated with lower likelihood to be listed for and receive transplantation. ${ }^{69}$

The association between low health literacy and decreased access to transplant is concerning, due to transplantation being a preferred treatment choice for many. ${ }^{70}$ As presented in the HeaL-T, diminished access and utilization of healthcare services as a result of low health literacy may influence, and be influenced by, provider-patient interactions and selfmanagement capacity. Ultimately, patient outcomes may be negatively affected.

\section{Self-management and adherence}

Self-management refers to the ability to complete daily healthcare tasks and adhere to the treatment regimen, which are necessary to ensure optimal health outcomes. ${ }^{71}$ This ability may be influenced by external factors (eg, health education, media, support technology, and other resources) and internal factors such as knowledge, skills and beliefs, problem-solving ability, motivation, participation in decisionmaking, and self-efficacy (Figure 1). ${ }^{15,16}$ Defined as an individual's belief in their capacity to perform behaviors needed to achieve specific goals, self-efficacy may be considered the foundation of self-management and is associated with SOTR self-management tasks such as physical activity and medication adherence..$^{43,72-77}$

Previous studies suggest patients with low health literacy suffer deficits in self-management and reduced medication adherence compared to patients with adequate health literacy. In their systematic review of health literacy in the general patient population, Berkman et al found that low health literacy was associated with reduced ability to 
understand/interpret labels and health messages as well as reduced ability to appropriately take medications. ${ }^{3}$ Similarly, Geboers et al found that self-management abilities were reduced in individuals with lower health literacy when controlled for age. ${ }^{78}$ Among 784 adults aged 55-74 years, Wolf et al found that low health literacy was associated with poor performance on healthcare tasks such as dosing and organizing medication and healthcare problem-solving. ${ }^{54}$

Disappointingly, research delving into the relationship between health literacy and self-management (other than medication adherence) in the transplant population was difficult to locate. However, as previously described, Jones et al found that patients wait-listed for renal transplantation with higher health literacy had increased understanding of the necessity of their posttransplant immunosuppression therapy as well as increased confidence about taking medications post-transplant - qualities necessary for proper self-management. ${ }^{49}$

\section{Medication adherence}

Medication adherence is the one aspect of posttransplant self-management for which considerable evidence exists regarding the relationship with health literacy, and therefore it is specifically displayed in the HeaL-T (Figure 1). Health literacy is consistently linked to adherence in general patient populations, and studies in the transplant population are consistent with these findings. ${ }^{3,58,79,80}$ Chisholm et al developed the Immunosuppressant Therapy Barrier Scale ${ }^{\odot}\left(\right.$ ITBS $^{\odot}$ ) to assess adherence barriers among SOTRs, and noted that increased barriers are associated with nonadherence. ${ }^{81}$ Several barriers enumerated in the $\operatorname{ITBS}^{\odot}$ reflect aspects of health literacy including understanding of why medications are needed and how and when to take medications. ${ }^{81}$ Serper et al found that lower health literacy in liver transplant recipients was associated with greater nonadherence to medication regimens which included immunosuppressants, other transplant-related medications, and medications to treat comorbidities. ${ }^{10}$ Serper et al also found that patients with low health literacy were more likely to report a medication tradeoff and, in turn, those with medication tradeoffs were more likely to report medication nonadherence. ${ }^{18}$ Demian et al reported similar results, noting that low health literacy was associated with medication nonadherence in kidney transplant recipients. ${ }^{17}$ These cumulative findings are troubling due to the relationship between medication nonadherence and negative health outcomes in transplant recipients, including increased graft rejection, graft failure, and mortality. ${ }^{4,8,82-85}$

\section{Healthcare provider-patient interaction}

As suggested in the HeaL-T (Figure 1), reciprocal relationships exist among access to care, self-management/ adherence, and healthcare provider-patient interactions. As with the first two healthcare-level factors, interactions and communication between healthcare providers and patients may be negatively impacted by low health literacy and vice versa, as interactions may in turn influence health literacy. Paasche-Orlow and Wolf noted that healthcare providers often do not use interviewing techniques designed for utilization with patients who have low levels of health literacy. ${ }^{15}$ As a result, providers may not deliver information and instructions in ways that patients can understand and process. Patients who do not fully understand healthcare information and instructions may not ask pertinent follow-up questions, appropriately complete self-management tasks, or be able to successfully navigate the healthcare system, among other consequences.

Interactions with patients may also be influenced by various provider characteristics such as communication style, experience level, and treatment milieu. As an example, provider communication that supports patient autonomy is associated with higher quality patient decisions (in which "higher quality" is defined by having adequate time, information, and involvement in decision-making, satisfaction, and no regrets with the decision). ${ }^{86}$ Another possible issue is racial/ethnic or gender concordance vs discordance between patient and practitioner. As mentioned previously, patients may prefer same-sex healthcare providers due to cultural or religious mores/tenets, or due to the sensitivity of the particular health issue (eg, female patients may prefer female providers when dealing with reproductive issues). Additionally, racial/ethnic concordance between patient and provider may promote trust and better communication, as providers "from racial and ethnic minority backgrounds may possess culturally specific knowledge, skills, and experience that reduce barriers" to the patient-provider relationship. ${ }^{87-90}$ When discordance exists, depending on the circumstances, patient health literacy may not be appropriately addressed.

The following section explores interventions, strategies, and tools that may improve the quality of provider-patient interactions and address the problem of low health literacy.

\section{Interventions and future developments}

Given the relationships noted in the HeaL-T between posttransplant outcomes and health literacy, the application of this model to develop strategies to optimize health outcomes 
may benefit care. As healthcare professionals, we are challenged to confront the limitations presented by low health literacy and implement strategies to support patients and caregivers through the transplant process, as well as through self-management tasks (eg, medication adherence) necessary for graft survival.

Health literacy of SOTRs and their caregivers should be assessed multiple times before and after transplant, given that literacy levels may vary according to the context or task the patient and/or caregiver is being asked to accomplish. Liang and Brach suggest healthcare providers adopt universal health literacy precautions such as providing easily understandable instructions, asking patients and caregivers to describe how they are going to follow treatment instructions, and offering help filling out healthcare forms.${ }^{91}$ Healthcare providers should also improve their own health literacy skills by learning how to identify patients and caregivers with low health literacy, participating in ongoing communication skills building, and utilizing educational strategies such as open-ended questions and Teach-Back in all patients with suspected low health literacy. ${ }^{91-94}$ Open-ended questions allow providers to explore patient understanding beyond simple "yes" or "no" answers. Teach-Back and similar educational methods are "a way of checking understanding by asking patients to state in their own words what they need to know or do about their health," how they should take their medications, and how they should perform various other tasks and behaviors related to treatment. These methods allow healthcare providers to confirm they have provided explanations and instructions in a manner that patients/caregivers understand, rather than place the onus of confirmation on the patient/caregiver. ${ }^{95}$

Education provided to patients and caregivers should be simple and easy to understand. ${ }^{91,96}$ Burke et al suggested that potential transplant candidate education should begin in the primary care setting and be tailored to address the individual's health literacy level. ${ }^{97}$ Written materials should avoid medical jargon, be at or below the fifth-grade reading level, presented in large font size, culturally sensitive, and presented in small increments (eg, 3-5 information points at a time). ${ }^{9,22,96,98}$ Alternate teaching methods/tools might also be useful for individuals with low health literacy such as flash cards, graphics/illustrations, QR-barcoded bottles, animated videos, and online patient portals via which patients can access health information and data, as well as any educational materials posted by providers. 9,22,91,94,99,100 Yeung et al examined the effects of a pharmacist-provided intervention in which patients with diabetes, heart failure, and/or hypertension were provided medication and disease education using flashcards written at a first-grade level or below (content also available as online videos using QR-coded medication bottles). ${ }^{99}$ The flashcards and videos were used to educate intervention patients on medication indications and administration, disease state, and common side effects. The investigators found that following the intervention, patients in the intervention group had significantly greater medication adherence than patients in the control group. ${ }^{99}$

The use of technology such as computers, smartphones, text messaging, social media platforms, self-management apps, and other technological tools has revolutionized how we learn and communicate, and can provide enormous support to individuals with low health literacy. ${ }^{101}$ McGillicuddy et al designed and implemented a mobile health intervention, consisting of electronic medication tray (which emitted reminder signals) and text message or email reminders, to improve medication adherence among hypertensive renal transplant recipients. ${ }^{102}$ They found that the intervention group experienced improved adherence and blood pressure control compared to the control group. ${ }^{102}$ Gordon et al designed a culturally targeted website regarding living kidney donation and transplantation for use among Hispanics/ Latinos, and found that website exposure was associated with increased knowledge scores. ${ }^{103,104}$ Website content was written at a 5 th to 8 th grade level and included videos, photographs, factsheets, games, and interactive graphics. In another example, Lam et al conducted a study in which hypertensive patients served by community pharmacies were provided audio-assisted medication instructions in the form of "Talking Pill Bottles."105 The talking pill bottle allowed pharmacists to record patient-centered counseling messages and instructions that patients could access at any time for guidance on how to take medications. Patients in the intervention arm experienced significantly decreased blood pressure, suggesting this technology can positively impact patient outcomes. ${ }^{105}$

Although promising, the literature concerning technological interventions to address low health literacy suggests some of these strategies are not more effective than standard care. For example, Harrison et al performed a randomizedcontrolled trial examining a computer-based education program vs standard teaching methods in SOTRs. ${ }^{106}$ They found there was no difference in knowledge improvement between the intervention and control groups; however, patient satisfaction did increase in the computer-based education arm. ${ }^{106}$ Future research should further evaluate the utility of talking pill bottles, text messaging, and similar technologies across SOTR groups to mitigate low health literacy and improve 
Table 2 Intervention strategies, tools, and resources to address low health literacy,22,91-105,108-110

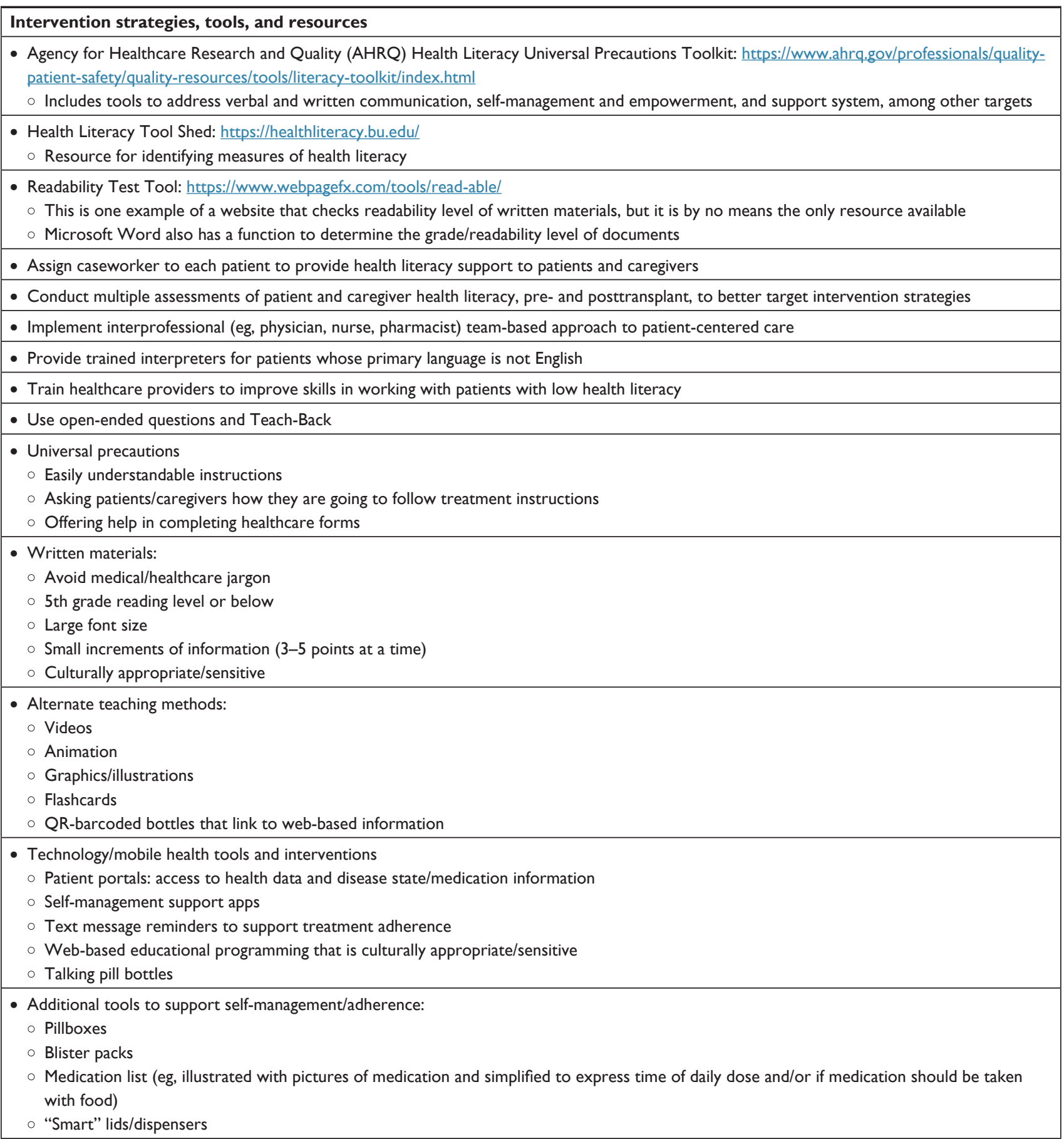

healthcare access, utilization, self-management/adherence, and ultimately health outcomes. As Bonner et al found that younger chronic kidney patients ( $<60$ years old) were more likely than older patients to use internet and mobile phone technology to obtain information about healthcare, research aimed at helping aging populations efficiently use technological interventions would also be advisable. ${ }^{107}$
Refer to Table 2 for additional information concerning intervention strategies, tools, and resources for working with SOTRs and caregivers with low health literacy., ${ }^{9,22,91-105,108-110}$

\section{Conclusion}

In summary, the HeaL-T is a framework for facilitating understanding of the multitude complex factors associated 
with health literacy in the solid-organ transplant population. Low health literacy increases the likelihood of negative health outcomes among SOTRs, and therefore it is a critical consideration in the care of this population. Health literacy is associated with a number of patient-level factors (eg, demographics, culture, socioeconomic status, cognitive function), healthcare-level factors (access, self-management, providerpatient interactions), and caregiver factors. Consideration of the influence of these various factors may better inform the design and implementation of interventions to address and mitigate the effects of low health literacy. Although some strategies are suggested herein, future research should explore the HeaL-T as a foundation to develop additional interventions to improve outcomes of SOTRs with low health literacy.

\section{Abbreviations}

ESL, English as a second language; HeaL-T, health literacy model in transplantation; IRR, incidence rate ratio; ITBS $^{\odot}$, Immunosuppressant Therapy Barrier Scale ${ }^{\circledR}$; NVS, Newest Vital Sign; REALM, Rapid Estimate of Adult Literacy in Medicine; RR, relative risk; SOTRs, solid-organ transplant recipients; TOFHLA, Test of Functional Health Literacy in Adults.

\section{Author contributions}

Dr Marie A Chisholm-Burns' specific contributions to this work include: 1) conception of the work; 2) writing and editing the manuscript; 3) final approval of the version to be published; and 4) agreement to be accountable for all aspects of the work in ensuring that questions related to the accuracy or integrity of any part of the work are appropriately investigated and resolved.

Dr Christina A Spivey's specific contributions to this work include: 1) conception of the work and participation in the literature search; 2) writing and editing the manuscript; 3) final approval of the version to be published; and 4) agreement to be accountable for all aspects of the work in ensuring that questions related to the accuracy or integrity of any part of the work are appropriately investigated and resolved.

Mr Logan R Pickett's specific contributions to this work include: 1) conception of the work and participation in the literature search; 2) writing and editing the manuscript; 3) final approval of the version to be published; and 4) agreement to be accountable for all aspects of the work in ensuring that questions related to the accuracy or integrity of any part of the work are appropriately investigated and resolved.

\section{Disclosure}

The authors report no conflicts of interest in this work.

\section{References}

1. Centers for Disease Control and Prevention. What is health literacy? Available from: https://www.cdc.gov/healthliteracy/learn/index.html. Accessed June 28, 2018.

2. World Health Organization. Track 2: health literacy and health behavior. Available from: http://www.who.int/healthpromotion/ conferences/7gchp/track2/en/. Accessed June 6, 2018.

3. Berkman ND, Sheridan SL, Donahue KE, Halpern DJ, Crotty K. Low health literacy and health outcomes: an updated systematic review. Ann Intern Med. 2011;155(2):97-107.

4. Chisholm MA, Fair J, Spivey CA. Health literacy and transplant patients and practitioners. Public Health. 2007;121(10):800-803.

5. Kutner M, Greenberg E, Jin Y, Paulsen C. The health literacy of America's adults: results from the 2003 National Assessment of Adult Literacy (NCES 2006-483). US Department of Education. Washington, DC: National Center for Education Statistics; 2006. Available from: https://nces.ed.gov/pubs2006/2006483.pdf. Accessed June 28, 2018.

6. Escobedo W, Weismuller P. Assessing health literacy in renal failure and kidney transplant patients. Prog Transplant. 2013;23(1):47-54.

7. Gordon EJ, Wolf MS. Health literacy skills of kidney transplant recipients. Prog Transplant. 2009;19(1):25-34

8. Taylor DM, Bradley JA, Bradley C, et al. ATTOM Investigators. Limited health literacy in advanced kidney disease. Kidney Int. 2016; 90:685-695.

9. Cajita MI, Denhaerynck K, Dobbels F, et al. Health literacy in heart transplantation: Prevalence, correlates and associations with health behaviors-Findings from the international BRIGHT study. $J$ Heart Lung Transplant. 2017;36(3):272-279.

10. Serper M, Patzer RE, Reese PP, et al. Medication misuse, nonadherence, and clinical outcomes among liver transplant recipients. Liver Transpl. 2015;21(1):22-28

11. Howard DH, Gazmararian J, Parker RM. The impact of low health literacy on the medical costs of Medicare managed care enrollees. Am J Med. 2005;118(4):371-377.

12. Friedland R. New estimates of the high costs of inadequate health literacy. In: Pfizer Inc. Proceedings of Pfizer Conference Promoting Health Literacy: A Call to Action, 7-8 October, 1998: 6-10. Washington, DC: Pfizer, Inc.

13. Weiss BD, Palmer R. Relationship between health care costs and very low literacy skills in a medically needy and indigent Medicaid population. J Am Board Fam Med. 2004;17(1):44-47.

14. Taylor DM, Fraser S, Dudley C, et al. Health literacy and patient outcomes in chronic kidney disease: a systematic review. Nephrol Dial Transplant. 2018;33:1545-1558.

15. Paasche-Orlow MK, Wolf MS. The causal pathways linking health literacy to health outcomes. Am J Health Behav. 2007;31(Suppl 1):19-26.

16. Dageforde LA, Cavanaugh KL. Health literacy: emerging evidence and applications in kidney disease care. Adv Chronic Kidney Dis. 2013; 20(4):311-319.

17. Demian MN, Shapiro RJ, Thornton WL. An observational study of health literacy and medication adherence in adult kidney transplant recipients. Clin Kidney J. 2016;9(6):858-865.

18. Serper M, Reese PP, Patzer RR, et al. The prevalence, risk factors, and outcomes of medication trade-offs in kidney and liver transplant recipients: a pilot study. Transpl Int. 2018;31:870-879.

19. Miller-Matero LR, Bryce K, Hyde-Nolan ME, et al. Health literacy status affects outcomes for patients referred for transplant. Psychosomatics. 2016;57(5):522-528.

20. Cavanaugh KL, Wingard RL, Hakim RM, et al. Low health literacy associates with increased mortality in ESRD. J Am Soc Nephrol. 2010; 21(11):1979-1985. 
21. Baker DW, Parker RM, Williams MV, Clark WS. Health literacy and the risk of hospital admission. J Gen Intern Med. 1998;13(12):791-798.

22. Dageforde LA, Petersen AW, Feurer ID, et al. Health literacy of living kidney donors and kidney transplant recipients. Transplantation. 2014; 98(1):88-93.

23. Levy H, Janke A. Health Literacy and Access to Care.J Health Commun. 2016;21(Suppl 1):43-50.

24. Cutilli CC, Simko LC, Colbert AM, Bennett IM. Health literacy, health disparities, and sources of health information in U.S. older adults. Orthop Nurs. 2018;37(1):54-65.

25. Toçi E, Burazeri G, Myftiu S, Sørensen K, Brand H, et al. Health literacy in a population-based sample of adult men and women in a South Eastern European country. J Public Health. 2016;38(1):6-13.

26. Alberti TL, Morris NJ. Health literacy in the urgent care setting: What factors impact consumer comprehension of health information? $J \mathrm{Am}$ Assoc Nurse Pract. 2017;29:242-247.

27. Miller-Matero LR, Hyde-Nolan ME, Eshelman A, Abouljoud M. Health literacy in patients referred for transplant: do patients have the capacity to understand? Clin Transplant. 2015;29(4):336-342.

28. Garcia CH, Espinoza SE, Lichtenstein M, Hazuda HP. Health literacy associations between Hispanic elderly patients and their caregivers. J Health Commun. 2013;18(Suppl 1):256-272.

29. Singleton K, Krause EMS. Understanding cultural and linguistic barriers to health literacy. OJIN. Online J Issues Nurs. 2009;14(3): Manuscript 4.

30. Christy SM, Gwede CK, Sutton SK, et al. Health Literacy among Medically Underserved: The Role of Demographic Factors, Social Influence, and Religious Beliefs. J Health Commun. 2017;22(11):923-931.

31. Neese B. Intercultural communication: high- and low-context cultures. Available from: https://online.seu.edu/high-and-low-context-cultures/. Accessed June 28, 2018.

32. Shaw SJ, Huebner C, Armin J, Orzech K, Orzech K, Vivian J. The role of culture in health literacy and chronic disease screening and management. J Immigr Minor Health. 2009;11(6):460-467.

33. Memon A, Taylor K, Mohebati LM, et al. Perceived barriers to accessing mental health services among black and minority ethnic (BME) communities: a qualitative study in Southeast England. BMJ Open. 2016;6(11):e012337.

34. Mcdonald JT, Kennedy S. Cervical cancer screening by immigrant and minority women in Canada. J Immigr Minor Health. 2007;9(4): 323-334.

35. Lofters A, Glazier RH, Agha MM, Creatore MI, Moineddin R. Inadequacy of cervical cancer screening among urban recent immigrants: a population-based study of physician and laboratory claims in Toronto, Canada. Prev Med. 2007;44(6):536-542.

36. Vahabi M, Lofters A. Muslim immigrant women's views on cervical cancer screening and HPV self-sampling in Ontario, Canada. BMC Public Health. 2016;16(1):868.

37. Brotto LA, Chou AY, Singh T, Woo JST, Jst W. Reproductive health practices among Indian, Indo-Canadian, Canadian East Asian, and EuroCanadian women: the role of acculturation. J Obstet Gynaecol Can. 2008;30(3):229-238.

38. Morgan M, Kenten C, Deedat S, Donate Programme Team. Attitudes to deceased organ donation and registration as a donor among minority ethnic groups in North America and the U.K.: a synthesis of quantitative and qualitative research. Ethn Health. 2013;18(4):367-390.

39. Jernigan M, Fahrenwald N, Harris R, Tsosie U, Baker LO, Buchwald D. Knowledge, beliefs, and behaviors regarding organ and tissue donation in selected tribal college communities. J Community Health. 2013;38(4): 734-740.

40. Lorini C, Ierardi F, Bachini L, et al. The antecedents and consequences of health literacy in an ecological perspective: results from an experimental analysis. Int J Environ Res Public Health. 2018;15(4): E798.

41. Rosario C, Modeste N, dos Santos H, et al. An examination of ecological predictors of health literacy in black college students. $J$ Am Coll Health. 2017;65(6):423-431.
42. Sarangarm D, Ernst A, Horner R, et al. Cross-sectional study of the relation of health literacy to primary language and emergency department length of stay. South Med J. 2017;110(12):796-801.

43. Mackey LM, Doody C, Werner EL, Fullen B. Self-management skills in chronic disease management: what role does health literacy have? Med Decis Making. 2016;36:741-759.

44. Kale MS, Federman AD, Krauskopf K, et al. The association of health literacy with illness and medication beliefs among patients with chronic obstructive pulmonary disease. PLoS One. 2015;10(4):e123937.

45. Federman AD, Wolf M, Sofianou A, et al. The association of health literacy with illness and medication beliefs among older adults with asthma. Patient Educ Couns. 2013;92(2):273-278.

46. Soones TN, Lin JL, Wolf MS, et al. Pathways linking health literacy, health beliefs, and cognition to medication adherence in older adults with asthma. J Allergy Clin Immunol. 2017;139(3):804-809.

47. Federman AD, Wisnivesky JP, Wolf MS, Leventhal H, Halm EA, et al. Inadequate health literacy is associated with suboptimal health beliefs in older asthmatics. $J$ Asthma. 2010;47(6):620-626.

48. Shiyanbola OO, Unni E, Huang YM, Lanier C. The association of health literacy with illness perceptions, medication beliefs, and medication adherence among individuals with type 2 diabetes. Res Social Adm Pharm. 2018;14:824-830.

49. Jones J, Rosaasen N, Taylor J, et al. Health literacy, knowledge, and patient satisfaction before kidney transplantation. Transplant Proc. 2016;48(8):2608-2614.

50. Speros CI. More than words: promoting health literacy in older adults. OJIN. Online J Issues Nurs. 2009;14(3):Manuscript 5.

51. Geboers B, Uiters E, Reijneveld SA, et al. Health literacy among older adults is associated with their 10-years' cognitive functioning and decline - the Doetinchem Cohort Study. BMC Geriatr. 2018; 18(1):77.

52. Harrington KF, Valerio MA. A conceptual model of verbal exchange health literacy. Patient Educ Couns. 2014;94(3):403-410.

53. Serper M, Patzer RE, Curtis LM, et al. Health literacy, cognitive ability, and functional health status among older adults. Health Serv Res. 2014;49(4):1249-1267.

54. Wolf MS, Curtis LM, Wilson EA, et al. Literacy, cognitive function, and health: results of the LitCog study. J Gen Intern Med. 2012;27(10): $1300-1307$.

55. Wallhagen MI, Strawbridge WJ, Shema SJ, Kurata J, Kaplan GA, et al. Comparative impact of hearing and vision impairment on subsequent functioning. J Am Geriatr Soc. 2001;49(8):1086-1092.

56. Bürker BS, Gude E, Gullestad L, et al. Cognitive function among longterm survivors of heart transplantation. Clin Transplant. 2017;31(12): e13143.

57. Cohen DG, Christie JD, Anderson BJ, et al. Cognitive function, mental health, and health-related quality of life after lung transplantation. Ann Am Thorac Soc. 2014;11(4):522-530.

58. Patzer RE, Serper M, Reese PP, et al. Medication understanding, non-adherence, and clinical outcomes among adult kidney transplant recipients. Clin Transplant. 2016;30(10):1294-1305.

59. Yuen EYN, Knight T, Ricciardelli LA, Burney S. Health literacy of caregivers of adult care recipients: A systematic scoping review. Health Soc Care Community. 2018;26(2):e191-e206.

60. Chisholm-Burns MA, Spivey CA, Wilks SE. Social support and immunosuppressant therapy adherence among adult renal transplant recipients. Clin Transplant. 2010;24(3):312-320.

61. Bevan JL, Pecchioni LL. Understanding the impact of family caregiver cancer literacy on patient health outcomes. Patient Educ Couns. 2008; 71(3):356-364.

62. Chen L, Xiao LD, de Bellis A. First-time stroke survivors and caregivers' perceptions of being engaged in rehabilitation. $J$ Adv Nurs. 2016;72(1):73-84.

63. Mullins J, Bliss DZ, Rolnick S, Henre CA, Jackson J. Barriers to communication with a healthcare provider and health literacy about incontinence among informal caregivers of individuals with dementia. J Wound Ostomy Continence Nurs. 2016;43(5):539-544. 
64. Pindus DM, Mullis R, Lim L, et al. Stroke survivors' and informal caregivers' experiences of primary care and community healthcare services - A systematic review and meta-ethnography. PLoS One. 2018; 13(2):e0192533.

65. della Pelle C, Orsatti V, Cipollone F, Cicolini G. Health literacy among caregivers of patients with heart failure: A multicentre cross-sectional survey. J Clin Nurs. 2018;27(3-4):859-865.

66. Yamashita T, Bardo AR, Millar RJ, Liu D. Numeracy and preventive health care service utilization among middle-aged and older adults in the U.S. Clin Gerontol. 2018;25:1-12.

67. Balakrishnan MP, Herndon JB, Zhang J, Payton T, Shuster J, Carden DL. The association of health literacy with preventable emergency department visits: a cross-sectional study. Acad Emerg Med. 2017;24(9): 1042-1050.

68. Grubbs V, Gregorich SE, Perez-Stable EJ, Hsu CY. Health literacy and access to kidney transplantation. Clin J Am Soc Nephrol. 2009; 4(1):195-200.

69. Kazley AS, Hund JJ, Simpson KN, Chavin K, Baliga P. Health literacy and kidney transplant outcomes. Prog Transplant. 2015;25(1):85-90

70. Schnuelle P, Lorenz D, Trede M, van der Woude FJ. Impact of renal cadaveric transplantation on survival in end-stage renal failure: evidence for reduced mortality risk compared with hemodialysis during long-term follow-up. J Am Soc Nephrol. 1998;9:2135-2141.

71. Grady PA, Gough LL. Self-management: a comprehensive approach to management of chronic conditions. Am J Public Health. 2014;104(8): e25-e31.

72. Carey MP, Forsyth AD. Teaching tip sheet: self-efficacy. American Psychological Association. Available from: http://www.apa.org/pi/ aids/resources/education/self-efficacy.aspx. Accessed June 28, 2018.

73. Weng LC, Yang YC, Huang HL, Chiang YJ, Tsai YH. Factors that determine self-reported immunosuppressant adherence in kidney transplant recipients: a correlational study. J Adv Nurs. 2017;73(1): 228-239.

74. Zelle DM, Corpeleijn E, Klaassen G, et al. Fear of movement and low self-efficacy are important barriers in physical activity after renal transplantation. PLoS One. 2016;11(2):e0147609.

75. Weng LC, Dai YT, Huang HL, Chiang YJ. Self-efficacy, self-care behaviours and quality of life of kidney transplant recipients. J Adv Nurs. 2010;66(4):828-838

76. Silva AN, Moratelli L, Tavares PL, et al. Self-efficacy beliefs, locus of control, religiosity and non-adherence to immunosuppressive medications in kidney transplant patients. Nephrology. 2016;21(11): 938-943.

77. Russell CL, Ashbaugh C, Peace L, et al. Time-in-a-bottle (TIAB): a longitudinal, correlational study of patterns, potential predictors, and outcomes of immunosuppressive medication adherence in adult kidney transplant recipients. Clin Transplant. 2013;27(5):E580-E590.

78. Geboers B, de Winter AF, Spoorenberg SL, Wynia K, Reijneveld SA. The association between health literacy and self-management abilities in adults aged 75 and older, and its moderators. Qual Life Res. 2016; 25(11):2869-2877.

79. Song S, Lee SM, Jang S, et al. Mediation effects of medication information processing and adherence on association between health literacy and quality of life. BMC Health Serv Res. 2017;17(1):661.

80. Ahn YH, Ham OK. Factors associated with medication adherence among medical-aid beneficiaries with hypertension. West J Nurs Res. 2016;38(10):1298-1312.

81. Chisholm MA, Lance CE, Williamson GM, Mulloy LL. Development and validation of an immunosuppressant therapy adherence barrier instrument. Nephrol Dial Transplant. 2005;20(1):181-188.

82. Gaston RS, Hudson SL, Ward M, Jones P, Macon R. Late renal allograft loss: noncompliance masquerading as chronic rejection. Transplant Proc. 1999;31(4A):21S-23S.

83. Butler JA, Roderick P, Mullee M, Mason JC, Peveler RC, et al Frequency and impact of nonadherence to immunosuppressants after renal transplantation: a systematic review. Transplantation. 2004;77(5): 769-776.
84. Chisholm MA, Kwong WJ, Spivey CA. Associations of characteristics of renal transplant recipients with clinicians' perceptions of adherence to immunosuppressant therapy. Transplantation. 2007;84(9): 1145-1150.

85. Spivey CA, Chisholm-Burns MA, Damadzadeh B, Billheimer D. Determining the effect of immunosuppressant adherence on graft failure risk among renal transplant recipients. Clin Transplant. 2014; 28(1):96-104.

86. Martinez KA, Resnicow K, Williams GC, et al. Does physician communication style impact patient report of decision quality for breast cancer treatment? Patient Educ Couns. 2016;99(12):1947-1954.

87. Traylor AH, Schmittdiel JA, Uratsu CS, Mangione CM, Subramanian U. Adherence to cardiovascular disease medications: does patient-provider race/ethnicity and language concordance matter? J Gen Intern Med. 2010;25(11):1172-1177.

88. Cooper LA, Roter DL, Johnson RL, et al. Patient-centered communication, ratings of care, and concordance of patient and physician race. Ann Intern Med. 2003;139(11):907-915.

89. Blanchard J, Nayar S, Lurie N. Patient-provider and patient-staff racial concordance and perceptions of mistreatment in the health care setting. J Gen Intern Med. 2007;22(8):1184-1189.

90. Saha S, Shipman SA. The rationale for diversity in the health professions: a review of the evidence. US Department of Health and Human Services; October 2006. Available from: https://www.pipelineeffect. com/wp-content/uploads/2015/04/diversityreviewevidence.pdf. Accessed 26 September 2018.

91. Liang L, Brach C. Health literacy universal precautions are still a distant dream: analysis of U.S. data on health literate practices. Health Lit Res Pract. 2017;1(4):e216-e230.

92. Wittenberg E, Ferrell B, Kanter E, Buller H. Health Literacy: Exploring Nursing Challenges to Providing Support and Understanding. Clin J Oncol Nurs. 2018;22(1):53-61.

93. Coleman CA, Fromer A. A health literacy training intervention for physicians and other health professionals. Fam Med. 2015;47:388-392.

94. Berkman ND, Sheridan SL, Donahue KE, et al. Health literacy interventions and outcomes: an updated systematic review. Evid Rep Technol Assess. 2011;199:1-941.

95. Agency for Health Research \& Quality. Health literacy universal precautions toolkit, 2nd edition. Use the teach-back method: tool \#5. Available from: https:/www.ahrq.gov/professionals/quality-patientsafety/quality-resources/tools/literacy-toolkit/healthlittoolkit2-tool5. html. Accessed 2018 June 28.

96. Meppelink CS, Smit EG, Buurman BM, van Weert JC. Should we be afraid of simple messages? The effects of text difficulty and illustrations in people with low or high health literacy. Health Commun. 2015; 30(12):1181-1189.

97. Burke MT, Kapojos J, Sammartino C, Gray NA. Kidney disease health literacy among new patients referred to a nephrology outpatient clinic. Intern Med J. 2014;44(11):1080-1086.

98. Waterman AD, Mcsorley AM, Peipert JD, et al. Explore Transplant at Home: a randomized control trial of an educational intervention to increase transplant knowledge for Black and White socioeconomically disadvantaged dialysis patients. BMC Nephrol. 2015;16(1):150.

99. Yeung DL, Alvarez KS, Quinones ME, et al. Low-health literacy flashcards \& mobile video reinforcement to improve medication adherence in patients on oral diabetes, heart failure, and hypertension medications. J Am Pharm Assoc. 2017;57(1):30-37.

100. Rosaasen N, Mainra R, Kukha-Bryson A, et al. Development of a patient-centered video series to improve education before kidney transplantation. Patient Educ Couns. 2018;101:1624-1629.

101. Bergner EM, Nelson LA, Rothman RL, Mayberry L. Text messaging may engage and benefit adults with type 2 diabetes regardless of health literacy status. Health Lit Res Pract. 2017;1(4):e192-e202.

102. Mcgillicuddy JW, Gregoski MJ, Weiland AK, et al. Mobile health medication adherence and blood pressure control in renal transplant recipients: a proof-of-concept randomized controlled trial. JMIR Res Protoc. 2013;2(2):e32. 
103. Gordon EJ, Feinglass J, Carney P, et al. A culturally targeted website for Hispanics/Latinos about living kidney donation and transplantation: a randomized controlled trial of increased knowledge. Transplantation. 2016;100:1149-1160.

104. Gordon EJ, Feinglass J, Carney P, et al. A website intervention to increase knowledge about living kidney donation and transplantation among Hispanic/Latino dialysis patients. Prog Transplant. 2016; 26(1):82-91

105. Lam AY, Nguyen JK, Parks JJ, Morisky DE, Berry DL, Wolpin SE. Addressing low health literacy with "Talking Pill Bottles": A pilot study in a community pharmacy setting. J Am Pharm Assoc. 2017;57(1): 20-29.

106. Harrison JJ, Badr S, Hamandi B, Kim SJ. Randomized controlled trial of a computer-based education program in the home for solid organ transplant recipients: impact on medication knowledge, satisfaction, and adherence. Transplantation. 2017;101:1336-1343.
107. Bonner A, Gillespie K, Campbell KL, et al. Evaluating the prevalence and opportunity for technology use in chronic kidney disease patients: a cross-sectional study. BMC Nephrol. 2018;19(1):28.

108. Agency for Healthcare Research and Quality (AHRQ). AHRQ health literacy universal precautions toolkit, second edition. August 2018. Available from: https://www.ahrq.gov/professionals/quality-patientsafety/quality-resources/tools/literacy-toolkit/index.html. Accessed October 26, 2018.

109. Health Literacy Tool Shed. Available from: https://healthliteracy. bu.edu/. Accessed October 26, 2018.

110. Readability Test Tool. Available from: https://www.webpagefx.com/ tools/read-able/. Accessed October 26, 2018.

\section{Publish your work in this journal}

Patient Preference and Adherence is an international, peer-reviewed, open access journal that focuses on the growing importance of patient preference and adherence throughout the therapeutic continuum. Patient satisfaction, acceptability, quality of life, compliance, persistence and their role in developing new therapeutic modalities and compounds to optimize clinical outcomes for existing disease states are major areas of interest for the journal. This journal has been accepted for indexing on PubMed Central The manuscript management system is completely online and includes a very quick and fair peer-review system, which is all easy to use. Visit http://www dovepress.com/testimonials.php to read real quotes from published authors. 